[Article]

www.whxb.pku.edu.cn

\title{
一种新型的氢键自组装液晶光控取向膜
}

\author{
吕凤珍 ${ }^{1,2}$ 彭增辉 ${ }^{1}$ 张伶莉 ${ }^{3}$ 姚丽双 ${ }^{1,2}$ 刘 艳 ${ }^{1,2}$ 宣 丽 ${ }^{1, *}$ \\ ( 中国科学院长春光学精密机械与物理研究所, 应用光学国家重点实验室, 长春 130033 ; \\ 2 中国科学院研究生院, 北京 $100039 ;{ }^{3}$ 哈尔滨工业大学理学院物理系, 哈尔滨 150001)
}

\begin{abstract}
摘要：报道了一种新型的以氢键为驱动力的液晶自组装光控取向膜, 研究了薄膜的制备方法与光敏特性. 通过 聚(4-乙烯基吡啶)中的吡啶基团与光敏聚丙烯酰氧基肉桂酸间的氢键作用制备了 LBL(layer-by-layer)型的自组 装多层膜, 制备过程的紫外-可见光谱表明, 该组装过程为逐层、均匀沉积过程. 傅里叶变换红外光谱表明, 多层 膜的成膜驱动力为氢键. 用线性偏振紫外光辐照该薄膜, 多层膜中与光矢量方向匹配的光敏基团发生 [2+2]环加 成反应, 形成表面张力各向异性的薄膜. 用该薄膜作为向列相液晶的取向膜制成平行液晶器件, 在偏光显微镜下 观察, 发现获得了均一、稳定的取向效果.
\end{abstract}

关键词: 液晶; 光控取向; 自组装多层膜; 线性偏振紫外光; 氢键 中图分类号: 0644,0648

\section{A New Type of Hydrogen-Bonded LBL Photoalignment Film for Liquid Crystal}

\author{
LÜ Feng-Zhen ${ }^{1,2} \quad$ PENG Zeng-Hui ${ }^{1} \quad$ ZHANG Ling-Li ${ }^{3} \quad$ YAO Li-Shuang ${ }^{1,2}$ \\ LIU Yan ${ }^{1,2} \quad$ XUAN $\mathrm{Li}^{1, *}$
}

('State Key Laboratory of Applied Optics, Changchun Institute of Optics, Fine Mechanics and Physics, Chinese Academy of Sciences, Changchun 130033, P. R. China; ${ }^{2}$ Graduate School of the Chinese Academy of Sciences, Beijing 100039, P. R. China; ${ }^{3}$ Department of Physics, College of Science, Harbin Institute of Technology, Harbin 150001, P. R. China)

\begin{abstract}
A new type of hydrogen-bonded layer-by-layer (LBL) photoalignment film was fabricated and studied. The photosensitive hydrogen donor containing cinnamoyl groups (Poly (4-acryloyloxy)-cinnamic acid, PCA) was selfassembled with hydrogen accepter poly (4-vinylpyridine) (PVPy) in an organic solvent. Then a hydrogen-bondingdirected ultrathin film was formed. The uniformity and linear growth of the deposition procedure was proven by UVVis spectra and the interaction between PVPy and PCA was identified as hydrogen bonding using FT-IR spectroscopy. When linearly polarized ultraviolet light (LPUVL) irradiated the multilayer film, only the photosensitive double bond parallel to the polarization direction of LPUVL could be polymerized via [2+2] cycloaddition. After the irradiation an anisotropic film was prepared, and we used this anisotropic film as an alignment layer of nematic liquid crystals, and obtained uniform and stable alignment results.
\end{abstract}

Key Words : Liquid crystal; Photoalignment; Self-assembled multilayer; Linearly polarized ultra-violet light; Hydrogen-bond

液晶的表面取向技术是液晶显示器件的基础, 它直接决定着显示器件内部液晶分子的排列好坏, 是影响器件对比度、显示品质等性能的重要因素. 液
晶显示器产业化以来, 大部分采用摩擦法进行取向 处理 ${ }^{[1-3]} .1996$ 年 Schadt 等 ${ }^{[4]}$ 提出了液晶的光控取向 方法, 即偏振紫外光照射光敏性薄膜后得到的各向

Received: September 5, 2008; Revised: October 27, 2008; Published on Web: December 16, 2008.

*Corresponding author. Email: xuanli@ciomp.ac.cn; Tel:+86431-86176016; Fax:+86431-86176316.

国家自然科学基金(60736042, 60578035, 50703039)和吉林省与中国科学院科技合作项目(2008YHZ0005)资助项目

C Editorial office of Acta Physico-Chimica Sinica 
异性薄膜可以诱导液晶取向. 由于光控取向是一种 完全非接触式的液晶取向方法, 它克服了接触式摩 擦取向法的不足, 使器件制作更加简便、高效, 成为 20 世纪 90 年代以来液晶显示领域的研究热点 ${ }^{[4,5]}$.

传统的光控取向方法多采用匀胶机旋涂的方法 成膜 ${ }^{[4]}$. 光控取向材料 ${ }^{[6-12]}$ 多为高分子, 旋涂成膜后光 敏材料随机地分布在待反应薄膜中, 在进行偏振光 化学反应时, 光敏基团受到高分子主链的空间位阻 作用, 使反应程度降低, 取向效果和取向的长期稳定 性不理想. 2000年, Naciri等 ${ }^{[13]}$ 制备了含有肉桂酸基 团的自组装单层膜, 并作为光控取向膜取向向列相 液晶, 取得了很好的取向效果. 这主要是因为自组装 膜内的分子具有更高的有序性, 使偏振光化学反应 进行得更为完全、高效. 自组装多层膜也是一种有序 膜, 与自组装单层膜相比, 有着膜厚可控、较少缺陷 等优点 ${ }^{[14-16}$. 彭增辉等人 ${ }^{[17-19]}$ 利用聚电解质的静电沉 积技术在基板表面制备了含有肉桂酸基团的自组装 多层膜, 获得了很好的液晶取向效果. 聚电解质交替 沉积技术的应用环境是在水溶液中, 这给许多不溶 于水的有机功能分子的组装带来很大的困难. 目前, 由Zhang ${ }^{[20]}$ 和Rubner ${ }^{[2] 1}$ 等人分别报道的以氢键为驱 动力的自组装多层膜技术引起了大家的广泛关注. 氢键在有机溶剂中比较容易形成, 这使一些不溶于 水或遇水分解的功能分子的交替组装成为可能, 在 很大程度上扩大了组装材料的应用范围. 本文把以 氢键为驱动力的自组装多层膜引人到液晶的光控取 向中, 设计合成了端基含有羧酸基团的光敏材料聚 丙烯酰氧基肉桂酸(PCA). 利用聚(4-乙烯基吡啶) (PVPy)中的吡啶基团与光敏材料 PCA(结构式如图 1)中的羧酸基团间的氢键作用制备了LBL型的多层 沉积膜. 把该氢键自组装多层膜用作液晶的光控取 向膜, 用线性偏振紫外光照射该组装多层膜, 与偏振 光偏振方向平行的光敏双键发生 $[2+2]$ 环加成反应, 形成具有共同取向方向的大分子, 从而使膜表面产 生表面张力的各向异性.

\section{1 实验部分}

\section{1 试剂及仪器}

3-氨丙基-三甲氧基硅烷、聚(4-乙烯基吡啶) (PVPy, $\left.M_{\mathrm{W}}=60000\right)$ 购自 Aldrich 公司, 未经纯化直接 使用; 4-羟基肉桂酸、枝化的聚亚乙基亚胺(PEI, $\left.M_{\mathrm{W}}=70000\right)$ 购自 Alfa 公司; 四氢呋喃(THF, 天津天 泰化学品公司)使用前经过如下处理: 加人一定量氢
化铝锂回流 $3 \mathrm{~h}$, 蒸馏收集 $66-67{ }^{\circ} \mathrm{C}$ 馏分; 二氧六环、 乙醇使用前重新蒸馏; 液晶材料为 $\mathrm{TEB} 30 \mathrm{~A}\left(T_{\mathrm{C}}=61.2\right.$ ${ }^{\circ} \mathrm{C}, T_{\mathrm{c}}$ 为液晶的清亮点温度. 石家庄永生华清液晶有 限公司).

紫外可见光谱仪为岛津公司 UV-3101PC 型; 辐 照用紫外光源为 $400 \mathrm{~W}$ 的 $\mathrm{Hg}-\mathrm{Xe}$ 灯, 其后放置 Glan-Talor 棱镜得到线性偏振紫外光, $\lambda=297 \mathrm{~nm}$ 处 光强为 $0.30 \mathrm{~mW} \cdot \mathrm{cm}^{-2}$; Bio-Rad 公司 FTS3000 型红 外光谱仪; 新天公司偏光显微镜, 上下两个偏振片 的偏振方向正交; 液晶器件的性能参数采用北方液 晶中心生产的 LCT-5016C 型液晶综合测试仪测定.

\section{2 光敏材料(聚丙烯酰氧基肉桂酸(PCA))的制备}

取 4-羟基肉桂酸 $13.12 \mathrm{~g}(80 \mathrm{mmol})$ 、氢氧化钠 $6.4 \mathrm{~g}(160 \mathrm{mmol})$ 溶于 $40 \mathrm{~mL}$ 水中, 再加人 $40 \mathrm{~mL}$ 二 氧六环搅拌均匀. 控温在 $5-10{ }^{\circ} \mathrm{C}$, 缓慢滴加丙烯酰 氯 $9.8 \mathrm{~mL}\left(\rho=1.11 \mathrm{~g} \cdot \mathrm{mL}^{-1}, 120 \mathrm{mmol}\right)$ 滴加完成后室 温下摚拌反应 $4 \mathrm{~h}$. 反应完全后用稀盐酸中和反应 液, 产物以白色絮状沉淀析出, 过滤出沉淀, 分别用 温水、稀盐酸、水依次洗涤, 干燥, 用柱色谱分离得到 白色产物 $\mathbf{1}(11.3 \mathrm{~g}, 52 \mathrm{mmol})$, 产率 $65 \%$, 熔点 $186{ }^{\circ} \mathrm{C}$. $\operatorname{FTIR}\left(\right.$ 固体粉末, $\left.\mathrm{KBr}, \mathrm{cm}^{-1}\right)$ : $979(=\mathrm{CH}, \delta), 1169(\mathrm{C}-$ $\mathrm{O}, \nu), 1635(\mathrm{C}=\mathrm{C}, \nu) 1688(\mathrm{C}=\mathrm{O}, \nu), 1743(\mathrm{C}=\mathrm{O}, \nu)$.

取 $1.0 \mathrm{~g}$ 产物 $\mathbf{1}$, 溶于 $20 \mathrm{~mL}$ 氯仿中, 加入溶质 质量 $0.5 \%$ 的偶氮二异丁氧(AIBN)作为引发剂, 在氮 气保护下加热 $70{ }^{\circ} \mathrm{C}$ 回流聚合. 回流 $12 \mathrm{~h}$ 后, 反应液 变粘, 将反应液倒人乙醇中, 产物以沉淀析出, 抽滤 后用氯仿溶解, 再用无水乙醇沉淀, 抽滤后真空干 燥, 获得最终产物 PCA, 产率为 50\%. FTIR (固体粉 末, $\left.\mathrm{KBr}, \mathrm{cm}^{-1}\right): 1167(\mathrm{C}-\mathrm{O}, \nu), 1635(\mathrm{C}=\mathrm{C}, \nu), 1741$ $(\mathrm{C}=\mathrm{O}, \nu)$. 反应式如图 1 所示.

\section{3 基板的处理方法和多层膜的制备}

用石英和氟化钙作为氢键自组装多层膜构筑的 基底, 石英片用于紫外-可见光谱及组装液晶盒的实 验, 氟化钙基底用于傅里叶变换红外光谱的测试, 两 种基底在组装多层膜前需要进行表面氨基化的修 饰. 石英片 $(2.0 \mathrm{~cm} \times 2.5 \mathrm{~cm})$ 置于 Piranha 溶液(浓 $\mathrm{H}_{2} \mathrm{SO}_{4}$ 与 $30 \% \mathrm{H}_{2} \mathrm{O}_{2}$ 体积比为 7:3) 中加热煮沸至无气 泡溢出, 取出后用去离子水冲洗, $110{ }^{\circ} \mathrm{C}$ 烘干. 将处 理后的石英片用 3-氨丙基-三甲氧基硅烷的二甲苯 溶液处理 ${ }^{[13]}$, 再于甲苯、乙醇和水中依次超声清洗 1 $\min$, 氮气吹干, 得到氨基修饰的表面. 氟化钙片 (1.0 $\mathrm{cm} \times 1.5 \mathrm{~cm}$ ) 先用吡啶、氯仿、甲醇和水漂洗, 干燥后 在 $1.5 \mathrm{~g} \cdot \mathrm{L}^{-1} \mathrm{PEI}$ 中性水溶液中浸泡 $1 \mathrm{~h}$, 然后漂洗、 


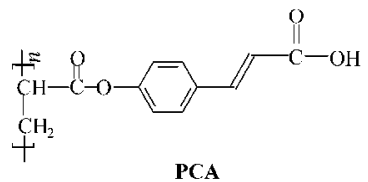<smiles>CCC(C)c1ccncc1</smiles>
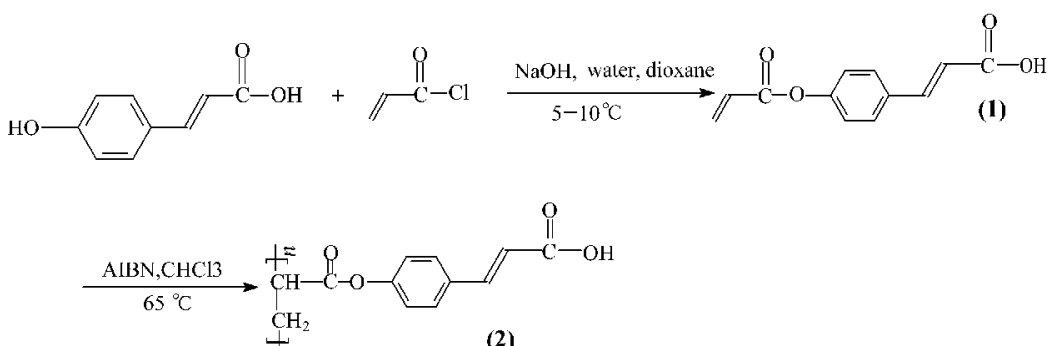

(2)

图 1 聚(4-乙烯基吡啶)(PVPy)与聚丙烯酰氧基肉桂酸(PCA)的化学结构式及 PCA 的合成路线

Fig.1 Chemical structures of poly(4-vinylpyridine) (PVPy) and poly(4-acryloyloxy)cinnamic acid (PCA), and synthetic route to $\mathrm{PCA}$

干燥备用. 氟化钙片表面被一层含有氨基的聚合物 (PEI)所修饰, 其表面由疏水变为亲水.

自组装多层膜的沉积过程如图 2 所示. 首先将 氨基修饰后的基片浸人到 PVPy 的乙醇溶液 ( $1 \mathrm{mg} \cdot$ $\mathrm{mL}^{-1}$ )中, $10 \mathrm{~min}$ 后取出,一层PVPy便沉积到基片表 面上, 即基片的表面被氢键的受体吡啶基团覆盖, 然 后用乙醇浸洗 3 次, 氮气吹干; 再将此基片浸人到 PCA的 THF溶液 $\left(1 \mathrm{mg} \cdot \mathrm{mL}^{-1}\right)$ 中 $10 \mathrm{~min}$, 一层PCA随 之沉积到基片上, 即基片的表面被氢键的给体羧酸 基团覆盖, 然后依次用 THF、乙醇淋洗, 氮气吹干. 重复上述操作得到 LBL 型的氢键自组装多层膜, 保 持膜的最外层为光敏 PCA 分子. 每一组 PVPy/PCA 记作一个膜层(bilayer).
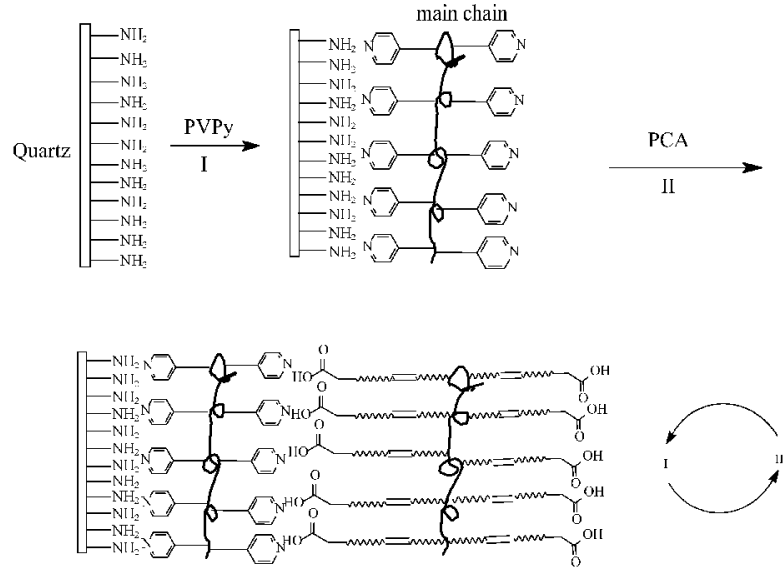

图 2 石英基板表面 PVPy/PCA 氢键自组装多层膜形成 过程示意图

Fig.2 Schematics of the layer-by-layer assembly of PVPy and PCA on a quartz substrate based on hydrogen bonding

(I) adsorption of PVPy and (II) adsorption of PCA

\section{4 液晶器件的制作}

将带有组装膜的基板用线性偏振紫外光辐照, 人射方向与基板表面垂直. 照射完成后将两片相同 的基板按照辐照紫外光偏振方向平行的方式组成液 晶盒, 基板间放置 $20 \mu \mathrm{m}$ 的隔垫物, $\mathrm{AB}$ 胶封盒. 然 后将向列相液晶 TEB30A 在各向同性状态下注人 液晶盒.

\section{2 结果与讨论}

\subsection{LBL 多层膜的成膜性能与光敏特性}

图3给出了构筑在氨基修饰的石英片上的氢键 自组装多层膜(PVPy/PCA)的 $1,2, \cdots, 9$ 层(bilayers)的 紫外-可见吸收光谱. 从谱图中可以看出, 由于吡啶

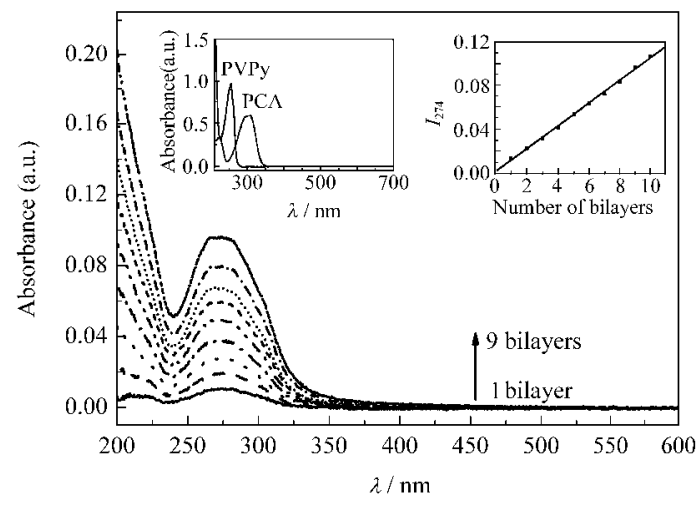

图 3 不同层数 PVPy/PCA 组装多层膜的紫外可见吸收光谱

Fig.3 UV-Vis absorption spectra of PVPy/PCA film on quartz substrate with increasing number of bilayers

The inset diagrams show the UV-Vis absorption spectra of PVPy and PCA, and the intensity of absorption at $274 \mathrm{~nm}$ with the number of bilayers 
基团和羧酸基团间的氢键连接, 使 PVPy 中吡啶环 在 $257 \mathrm{~nm}$ 的特征吸收峰与 PCA 中的光敏双键在 $300 \mathrm{~nm}$ 的特征吸收峰重叠(PVPy 和 PCA 的紫外吸 收谱图如图 3 中插图所示), 从而在 $274 \mathrm{~nm}$ 处形成 了一个特别宽的谱带. 图 3 中的插图给出了 $274 \mathrm{~nm}$ 处的吸收强度与多层膜沉积的双层数之间的依赖关 系, 即紫外吸收强度随多层膜的双层数的增加而线 性增加. 这充分说明 LBL 多层膜的形成过程中每层 沉积 PVPy 和 PCA 的量基本相同, 即氢键自组装多 层膜的生长是一个逐层、均匀沉积的过程.

图 4 是 10 层组装膜用线性偏振紫外光照射不 同时间的 UV-Vis 谱图. 从图中可以看出, $300 \mathrm{~nm}$ 处 的紫外谱峰随着光照时间的增加而不断下降, 表明 与苯环共轭的双键发生了聚合反应, 导致吸收强度 降低. Egerton 等 ${ }^{[22]}$ 认为肉桂酰基受到紫外光辐照发 生的反应为 $[2+2]$ 环加成反应, 同时伴有少量的 E-Z 异构反应. 对于 [2+2]周环反应, 两个位置临近的双 键吸收紫外光发生反应的过程和被吸收光的偏振态 有相关性, 只有相互匹配时才可能发生周环反应 ${ }^{[n]}$. 实验中用线性偏振紫外光辐照多层膜, 光化学反应 发生在紫外光的偏振方向上, 反应产物也沿着该方 向分布, 这样就得到了表面张力各向异性的薄膜. 从 图中可以看到, 辐照时间大于 $20 \mathrm{~min}$ 时, 随着光照 时间的增加, $300 \mathrm{~nm}$ 处的吸收峰强度基本不再发生 变化, 说明光敏双键已经基本反应完全. 从紫外谱图 中还可以发现, 随着 $300 \mathrm{~nm}$ 处吸收峰强度的降低, $257 \mathrm{~nm}$ 处吡啶环的特征吸收峰逐渐显现出来, 且由 于两个特征峰的相互影响, $257 \mathrm{~nm}$ 处的特征吸收峰 强度会随着 $300 \mathrm{~nm}$ 处紫外吸收峰的变化而变化.

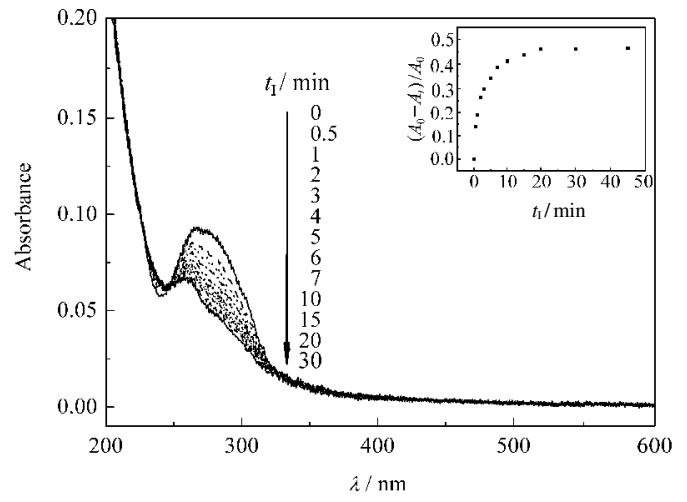

图 4 不同光照时间 $\left(t_{1}\right)$ 下 PVPy/PCA 组装多层膜的 紫外吸收光谱

Fig.4 UV-Vis spectra of a 10-bilayers PVPy/PCA film irradiated with different times $\left(t_{\mathrm{I}}\right)$

The inset diagram shows the change ratio in absorption at $300 \mathrm{~nm}$ as a function of LPUVL irradiation time
自组装膜在线性偏振紫外光辐照下, 光敏双键反应 的比率表示为 $\left(A_{0}-A_{t}\right) / A_{0}, A_{0}$ 和 $A_{t}$ 分别表示辐照时间 为 $0 \mathrm{~min}$ 和辐照时间为 $t \mathrm{~min}$ 时 $300 \mathrm{~nm}$ 处光敏双键 的吸收强度. 从图中可以看出, 随着光照时间的增 加, 光敏双键反应的比率迅速增大, 光照 $20 \mathrm{~min}$ 后 反应趋于平衡, 反应比率可以达到 $50 \%$.

\section{$2.2 \mathrm{PVPy} / \mathrm{PCA}$ 氢键多层膜成膜驱动力的傅里叶}

\section{变换红外光谱表征}

PVPy/PCA自组装多层膜的成膜驱动力可以用 傅里叶变换红外光谱进行表征. 图 5(a)为PCA 和 PVPy的红外光谱( $\mathrm{KBr}$ 压片), 图 5(b)为氟化钙基片上 组装 50 个PVPy/PCA双层膜的FTIR光谱图. 从多层 膜的红外谱图中发现, 多层膜在 2530 和 $1945 \mathrm{~cm}^{-1}$ 出 现两个新的吸收峰, 它们可以指认为 $\mathrm{O}-\mathrm{H}$ 键的伸 缩振动, 这两个新吸收峰的出现充分地说明了PVPy 和PCA之间形成了氢键 ${ }^{[23]}$. 此外从图中可以看到, 吡 啶基团在 1595 、1556和 $1450 \mathrm{~cm}^{-1}$ 处的特征吸收峰, 以 及PCA在1300-1650 $\mathrm{cm}^{-1}$ 范围内的吸收峰位置没有 发生变化, 说明组装膜中的PCA及PVPy中的吡啶基 团没有发生电离 ${ }^{[24]}$, 同样说明这种组装多层膜是在有
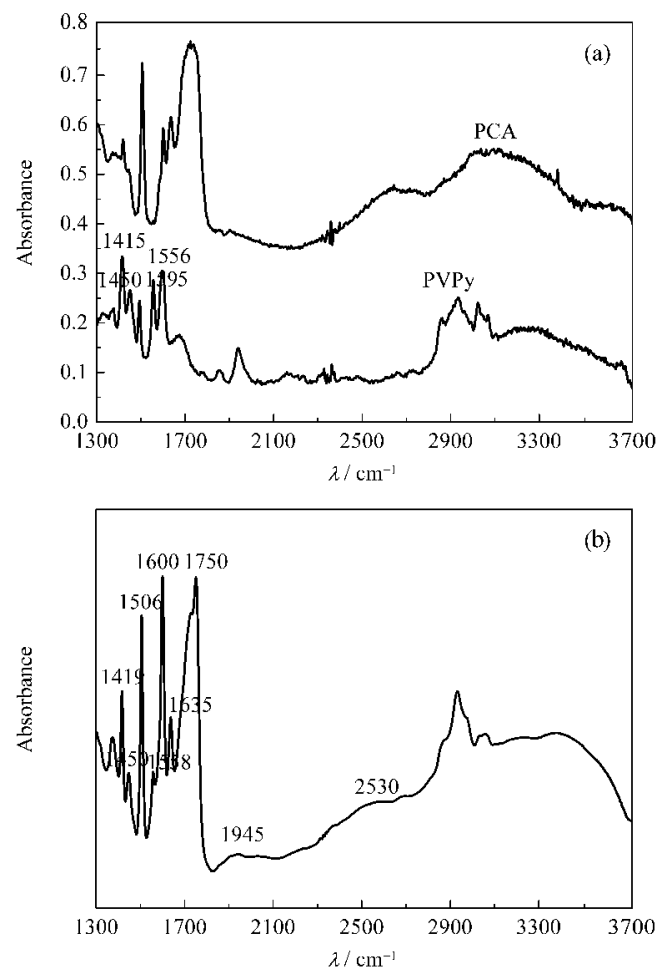

图 5 (a)PCA 和 PVPy 的红外光谱 $(\mathrm{KBr}$ 压片)和(b)氟化 钙基板表面 (PCA/PVPy $)_{50}$ 组装多层膜的红外光谱

Fig.5 IR spectra of (a) pure PCA and pure PVPy, and (b) a 50-bilayer PCA/PVPy assembled film on $\mathrm{CaF}_{2}$ plate 


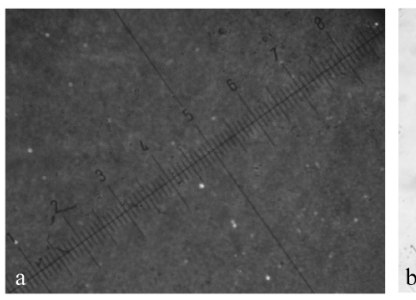

图 6 液晶器件亮暗态(a)和亮态(b)偏光显微镜照片

Fig.6 Photographs of dark (a) and bright (b) states of liquid crystal cell under polarized microscopy

机溶剂中进行的氢键自组装而非静电自组装.

\section{3 氢键多层膜取向液晶的能力}

将用偏振紫外光辐照后的带有 6 层组装膜的基 板做成平行液晶盒, 在正交偏光显微镜下观察液晶 在组装膜上的取向效果. 图 6 是偏光显微下该液晶 盒的亮暗态照片(放大 125 倍). 其中 (a)为辐照用紫 外光偏振片方向和显微镜起偏器方向平行时的暗态 照片, (b)为紫外光偏振方向和起偏器成 $45^{\circ}$ 角时的 亮态照片. 从图中可以看出, 用此种氢键多层膜作为 液晶取向膜时制成的器件中无取向不均匀引起的向 错线, 亮暗态都很均匀, 得到了很好的取向效果. 在 偏光显微镜下测定此液晶器件的静态对比度 $\left(I_{\text {max }}\right.$ I $\left.I_{\min }\right)$ 为 80 , 已接近于目前实用化器件的水平.

同时进行了未经光照氢键多层膜的取向实验. 石英基板表面形成多层膜后未经偏振紫外光照射, 直接组装成液晶盒灌注液晶, 在偏光显微镜下未观 察到明显的亮暗态, 而表现为一种多畴的花纹, 说明 多层膜对液晶的取向作用来源于偏振光照射时薄膜 内部的各向异性光化学反应. 液晶器件的使用有一 定的温度范围, 因此要求取向膜具备一定的热稳定 性. 实验中对器件进行了热稳定性测试, 将制成的器 件在 $100{ }^{\circ} \mathrm{C}$ 的烘箱中保持 $30 \mathrm{~min}$, 自然冷却后, 用 偏光显微镜观察发现取向效果仍然保持, 说明器件 的热稳定性良好. 此外, 用晶体旋转法测定 PVPy/ PCA 多层膜的预倾角较小, 大约为 $1^{\circ}$.

\section{3 结 论}

报道了一种新型的以氢键为驱动力的基于 PVPy/PCA 的 LBL 自组装液晶光控取向膜的制备 与光敏特性的研究. 傅里叶变换红外光谱证明多层 膜的成膜驱动力为氢键. 紫外-可见光谱表明氢键自 组装多层膜的生长是一个逐层、均匀沉积的过程, 且 经过线性偏振紫外光辐照后可以得到物理性质各向 异性的薄膜. 用该薄膜取向向列相液晶, 经过偏光显
微镜观察, 发现获得了均一、稳定的取向效果. 氢键 自组装多层膜技术使一些不溶于水或遇水分解的功 能分子的交替组装成为可能, 在很大程度上扩大了 组装材料的应用范围, 是一种颇具应用前景的液晶 自组装光控取向技术.

\section{References}

1 Cognard, J. Mol. Cryst. Liq. Cryst. Suppl. Ser., 1982, 1: 1

2 Toney, M. F.; Russell, T. P.; Logan, J. A.; Kikuchi, H.; Sands, J. M.; Kumar, S. K. Nature, 1995, 374: 709

3 Ichimura, K. Chem. Rev., 2000, 100(5): 1847

4 Schadt, M.; Seiberle, H.; Schuster, A. Nature, 1996, 381: 212

5 Seki, T.; Ichimura, K.; Fukuda, R.; Tanigaki, T.; Tamaki, T. Macromolecules, 1996, 29(3): 892

6 Ichimura, K.; Akita, Y.; Akiyama, H.; Kudo, K.; Hayashi, Y. Macromolecules, 1997, 30(4): 903

7 Jackson, P. O.; O'Neill, M.; Duffy, W. L.; Hindmarsh, P.; Kelly, S. M.; Owen, G. J. Chem. Mater., 2001, 13(2): 694

8 Ichimura, K.; Suzuki, Y.; Seki, T.; Hosoki, A.; Aoki, K. Langmuir, 1988, 4(5): 1214

9 Akiyama, H.; Momose, M.; Ichimura, K.; Yamamura, S. Macromolecules, 1995, 28(1): 288

10 Park, M. K.; Advincula, R. C. Langmuir, 2002, 18(11): 4532

11 Wang, Y. H.; Xu, C. Y.; Kanazawa, A.; Shiono, T.; Ikeda, T.; Matsuki, Y.; Takeuchi, Y. J. Appl. Phys., 1998, 84(1): 181

12 Wang, Y. H.; Xu, C. Y.; Kanazawa, A. J. Appl. Phys., 1998, 84(8): 4573

13 Naciri, J.; Fang, J. Y.; Moore, M.; Shenoy, D.; Dulcey, C. S.; Shashidhar, R. Chem. Mater., 2000, 12(11): 3288

14 Zhang, X.; Shen, J. C. Adv. Mater., 1999, 11(13): 1139

15 Wu, T.; Zhang, X. Chem. J. Chin. Univ., 2001, 22(6): 1057 [吴 涛, 张 希. 高等学校化学学报, 2001, 22(6): 1057]

16 Mao, G.; Tsao, Y.; Tirrell, M.; Davis, H. T.; Hessel, V.; Ringsdorf, H. Langmuir, 1993, 9(12): 3461

17 Peng, Z. H.; Xuan, L. Liquid Crystals, 2005, 32(2): 239

18 Peng, Z. H.; Yu, T.; Ruan, S. P.; Xuan, L. Chem. J. Chin. Univ., 2005, 26(1): 184 [彭增辉, 于 涛, 阮圣平, 宣 丽. 高等学校 化学学报, 2005, 26(1): 184]

19 Zhang, L. L.; Peng, Z. H.; Yao, L. S.; Fei, C. H.; Lv, F. Z.; Xuan, L. Applied Surface Science, 2007, 253: 3372

20 Wang, L. Y.; Wang, Z. Q.; Zhang, X.; Shen, J. C.; Chi, L. F. Macromol. Rapid. Commun., 1997, 18: 509

21 Stockton, W. B.; Rubner, M. F. Macromolecules, 1997, 30(9): 2717

22 Egerton, P. L.; Pitts, E.; Reisner, A. Macromolecules, 1981, 14(1): 95

23 Katim, T.; Kihara, H.; Uryu, T.; Fujishima, A.; Fréchet, J. M. J. Macromolecules, 1992, 25(25): 6836

24 Zhang, H. Y.; Fu, Y.; Wang, D.; Wang, L. Y.; Wang, Z. Q.; Zhang, X. Langmuir, 2003, 19(20): 8497 